\title{
CORRECTION
}

Check for updates

Cite this: RSC Adv., 2020, 10, 4464

rsc.li/rsc-advances

\section{Correction: Solution growth and thermal treatment of crystals lead to two new forms of 2-((2,6- dimethylphenyl)amino)benzoic acid}

\author{
Rong Hu, ${ }^{a}$ Yunping Zhoujin, ${ }^{a}$ Meng Liu, ${ }^{a}$ Mingtao Zhang, ${ }^{b}$ Sean Parkin, ${ }^{d}$ \\ Panpan Zhou, ${ }^{\mathrm{c}}$ Jianzhi Wang, ${ }^{\mathrm{a}}$ Faquan $\mathrm{Yu}^{\star a}$ and Sihui Long ${ }^{\star a}$
}

Correction for 'Solution growth and thermal treatment of crystals lead to two new forms of 2-((2,6dimethylphenyl)amino)benzoic acid' by Rong Hu et al., RSC Adv., 2018, 8, 15459-15470.

The new form, i.e. form II of 2-((2,6-dimethylphenyl)amino)benzoic acid identified in the original article is the same form as the one reported by V. Dokorou et al. ${ }^{1}$ The crystal structure reported by Dokorou is incorrect as they mistakenly doubled the unit cell, leading us to believe we were reporting a new form.

The recently published Comment article ${ }^{2}$ highlights our error in reporting form II as new.

The Royal Society of Chemistry apologises for these errors and any consequent inconvenience to authors and readers.

\section{References}

1 V. Dokorou, D. Kovala-Demertzi, J. P. Jasinski, A. Galani and M. A. Demertzis, Helv. Chim. Acta, 2004, 87, 1940.

2 P. R. S. Salbego, T. Orlando and M. A. P. Martins, RSC Adv., 2019, 9, 28195-28198.

${ }^{a}$ Key Laboratory for Green Chemical Process of Ministry of Education, School of Chemical Engineering and Pharmacy, Wuhan Institute of Technology, 693 Xiongchu Road, Wuhan, Hubei 430073, China.E-mail: fyuwucn@gmail.com; sihuilong@wit.edu.cn; longsihui@yahoo.com; Tel: +8602787194980

${ }^{b}$ Computational Center for Molecular Science, College of Chemistry, Nankai University, Tianjin, China

'Department of Chemistry, Lanzhou University, Lanzhou, Gansu, China

${ }^{d}$ Department of Chemistry, University of Kentucky, Lexington, Kentucky, USA 